\title{
Use of salidroside in a lipopolysaccharide-induced periventricular leukomalacia model
}

\author{
Mustafa Dilek$^{1}$, Gokce Kaya Dincel ${ }^{1}$, Ayhan Cetinkaya ${ }^{2}$, Mervan Bekdas $^{1}$, Nimet Kabakus ${ }^{1}$ \\ ${ }^{1}$ Bolu Abant Izzet Baysal University, Faculty of Medicine, Department of Pediatrics, Bolu, Turkey \\ ${ }^{2}$ Bolu Abant Izzet Baysal University, Experimental Animal Application and Research Center, Bolu, Turkey
}

\section{ABSTRACT}

Aim: Research into the different treatment methods based on the intrauterine lipopolysaccharide (LPS)-induced periventricular leukomalacia (PVL) model, as one of the main causes of morbidity in preterm infants still continues to be relevant. The present study investigates the effect on PVL of salidroside obtained from Rhodiola Rosea (golden root, orpin rose), which is a plant with known for its medicinal qualities.

Method: To develop an induced PVL model, a 500 microgram $/ \mathrm{kg}$ dose of LPS (Escherichia coli, serotype 055:B5, Sigma) was applied to two pregnant rats intraperitoneally on day 18, day 19 and day 20 of gestation. One of the LPS applied rats was given $25 \mathrm{mg} / \mathrm{kg}$ salidroside $(250 \mathrm{mg}$ Rhodiola root extract capsules, which include $3 \mathrm{mg}$ salidroside) by oral gavage (LPS+Salidroside), and a physiological saline solution was given to the control group.

After delivery, 10 offspring of the LPS-applied mother, nine offspring of the LPS+Salidrosideapplied mother and seven offspring of the control mother were sacrificed on postnatal day 7 with ether anesthesia. The caspase enzyme located in apoptosis pathways of 10 percent neutral-buffered formalin fixed brain tissue was stained immunohistochemically, and apoptotic cells were counted.

Results: No statistically significant difference was noted between the LPS+Salidroside group and the control group, while a statistically significant difference was noted between the LPS and LPS+Salidroside groups. It was observed that salidroside reduced LPS induced apoptosis.

Conclusion: The intended experimental neuroprotective effect of salidroside usage was provided through the inhibition of apoptosis in a PVL-damaged brain.

Keywords: Salidroside; lipopolysaccharide; periventricular leukomalacia; rat model; neuroprotection. 
Corresponding Author:

Dr. Mustafa Dilek,

Department of Pediatrics, Bolu Abant Izzet Baysal

University Faculty of Medicine, 14280, Gölköy,

Bolu, Turkey

E-mail: mustafadilek@gmail.com

ORCID ID: https://orcid.org/0000-0002-3802-0336

Received 2018-08-03, Revisions 2018-09-04

Accepted 2018-09-14

Publication Date 2018-10-01

\section{Introduction}

Periventricular leukomalacia (PVL) is a significant cause of morbidity in preterm infants. Hypomyelination develops because of a failure of the myelin-forming mature oligodendrocytes as a result of precursor oligodendrocyte cell damage in PVL, which caused cystic lesions in white matter or diffuse involvement. The predisposing factors for PVL are hypoxia, ischemia and inflammation [1-3].

The medicinal properties of Rhodiola Rosea (golden root, orpin rose) have been known for centuries. It induces neurotransmitter activity (e.g. serotonin, dopamine, norepinephrine), and also has inflammatory and antioxidant characteristics. It has been reported to reduce inflammation in LPS-induced microglial cell damage, and so can be used for the treatment of neurodegenerative diseases [4]. The active component in the plant is salidroside.

The effect of salidroside on an intrauterine lipopolysaccharide (LPS) administrationinduced Periventricular leukomalacia (PVL) model, as a major cause of morbidity in preterm infants, was investigated.

\section{Methods}

This study was approved by the AIBU Animal Research Local Ethics Committee and carried out in the AIBU Experimental Animals Application and Research Center and AIBU
Faculty of Science and Literature Alper Karakaş Laboratory. Female Wistar rats were fed standard pellets and water and kept in standard cages at room temperature at 50-60 percent humidity in a 12 hour light/dark cycle. Three selected female mature rats in their estrous cycle, ascertained through a vaginal smear, were mated, and the mating day was accepted as day 0 of pregnancy. To develop an induced PVL model, a 500 microgram $/ \mathrm{kg}$ dose of intraperitoneal LPS (Escherichia coli, serotype $055: B 5$, Sigma) was applied to two pregnant rats on days 18,19 and 20 of gestation. Of the LPS-applied rats, one was given $25 \mathrm{mg} / \mathrm{kg}$ salidroside (250 mg Rhodiola root extract capsules, containing $3 \mathrm{mg}$ salidroside) by oral gavage (LPS+Salidroside), and a physiological saline solution was given to the control group.

After delivery, 10 offspring of the LPS-applied mother, nine offspring of the LPS+Salidrosideapplied mother and seven offspring of the control mother were terminated under ether anesthesia by brain fixation using 4 percent paraformaldehyde via a transcardiac perfusion on postnatal day 7. The caspase enzyme located in the apoptosis pathways of the 10 percent neutral-buffered formalin fixed brain tissue was stained immunohistochemically. All sections (at least four sections for each tissue) were analyzed and those with artefacts apparent only due to staining, were excluded from assessment. Apoptotic cells with positive stained active caspase-3 immunoreactivity were counted, and the mean apoptotic cell number was determined for each brain. All groups were compared with a Mann-Whitney U-test using a PASW Statistics 18 program.

\section{Results}

After delivery, 10 offspring of the LPS-applied mother, 9 offspring of the LPS+Salidroside 
applied mother and seven offspring of the control mother were included in the study. No statistically significant difference was identified between the LPS+Salidroside group and the control group, while a statistically significant difference was determined between the LPS and LPS+Salidroside groups. It was observed that salidroside reduced LPS-induced apoptosis (Figure 1). The histopathological study of apoptotic cells is given in Figure 2.

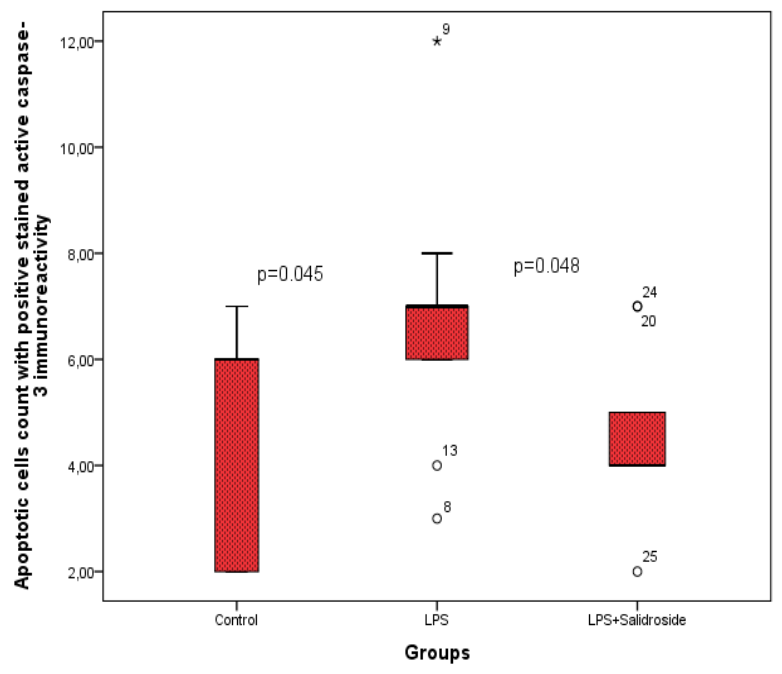

Figure 1. The apoptotic cell-count results in the groups.

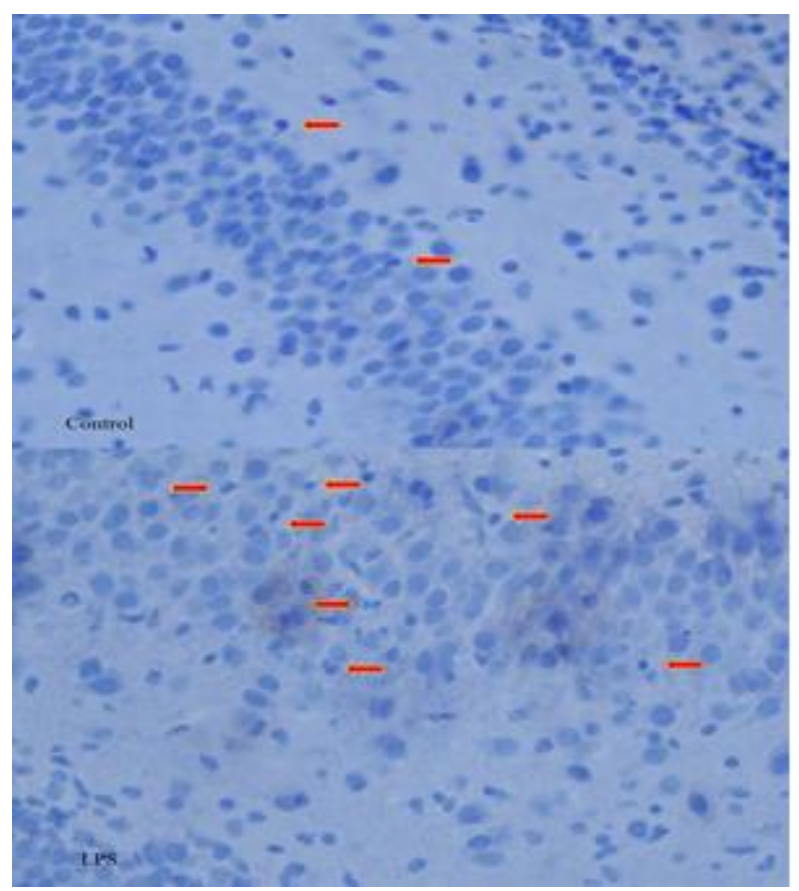

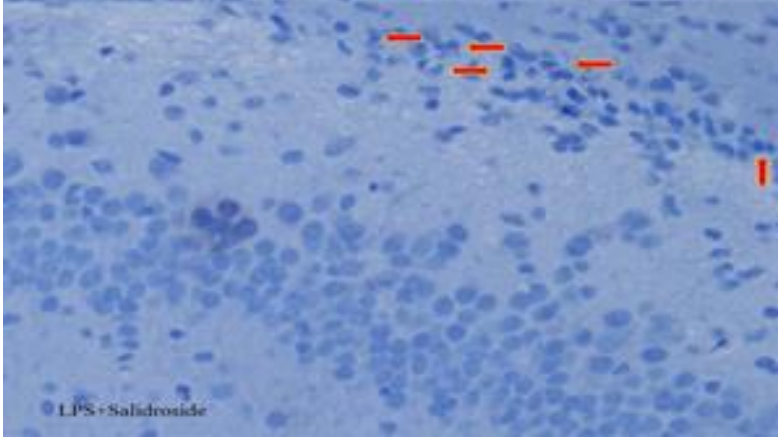

Figure 2. Representative light-microscopic images of anti-caspase-3 immunohistochemically labeled apoptotic cells (X100).

\section{Discussion}

The predisposing factors for PVL are hypoxia, ischemia and inflammation, while other possible mechanisms are microglial activation and axonal degeneration [1-3]. There are many factors that play a fundamental role in PVL pathogenesis, which include hypoxia, ischemia, both reactive oxygen (ROS) and nitrogen (RNS) free radical damage, and glutamate excitotoxicity. Myelination deficiencies related to the microglial activation and the loss of precursor oligodendrocytes were observed [5].

Hydroxyl radicals $\left(\mathrm{OH}^{-}\right)$, which are responsible for tissue damage, occur in two main ways in free radical damage, being formed by reactive oxygen species or by reactive nitrogen species. The pre-requirement for this is oxygen interacting with free electrons, and the formation of hydrogen peroxide and superoxide. Oxygen interacts with a free electron through the hypoxanthinexanthine oxidase-xanthine system in the reperfusion/reoxygenation phase after ischemia/hypoxia. Xanthine oxidase is an important source of free radicals, and it has been shown that Rhodiola Rosea inhibits xanthine oxidase enzyme activity [6]. In the same study, it was found that it also reduces 
oxidant enzyme activities such as lipoxygenase and tyrosinase. Salidroside, the active substance in Rhodiola Rosea, has also shown neuronal cell protective effects to glucose and serum-restriction in cell cultures [7]. Salidroside has also been reported to have neuroprotective effects in cases of glutamateinduced neuronal damage [8]. Salidroside has the potential to have a protective effect both on the reduction of oxidant and lytic enzyme activity and brain damage resulting from such excitotoxic agents as glutamate.

Salidroside causes the formation of dopaminergic neurons in mesenchymal stem cells, which increases the synthesis of such factors involved in neuronal development as BDNF, NGF and neurotrophin-3 [9]. It has been reported that antenatal neurotrophin exposure reduces hypomyelination in LPSinduced damage and has a protective effect against apoptosis [10]. Other studies have found that in the LPS-induced acute lung injury model in mice reduces the number of macrophages and neutrophils in broncho alveolar lavage and suppresses the production of such important inflammatory cytokines as TNF- $\alpha$, IL-6, IL-1 $\beta$ and NF- $\kappa$ B DNA-binding activation [11]. Intraperitoneal or intracervical injections of LPS into rats causes intrauterine inflammation, which leads to cytokine stimulation in the fetus and the neonatal brain, and results in white matter damage similar to PVL. Maternal LPS injections can have significant effects on myelination and astrogliosis in neonatal rat brains [12-14]. In the present study, its apoptosis-reducing effects and its effects in the LPS-induced PVL model have been observed. In other words, the intended experimental neuroprotective effect of Salidroside usage was provided with the inhibition of apoptosis in a PVL-damaged brain.
The antioxidant and anti-inflammatory features of salidroside mean that it may be neuroprotectant in PVL. It can also be suggested that positive results may be obtained by increasing the neuronal plasticity effect in developing brain tissue. Maternal salidroside administration may also be effective in preventing the intrauterine PVL process, although there have been no studies to date examining the effects of salidroside on brain damage in cases of periventricular leukomalacia. If its neuroprotective effects can be demonstrated in the periventricular leukomalacia process as a result of this study, an important step will be taken towards the prevention of periventricular leukomalacia, which is one of the most significant problems in premature babies.

\section{Compliance with ethical statements Conflicts of Interest: None.}

\section{Acknowledgments}

(This study was supported by Abant Izzet Baysal University - Scientific Research Projects Coordination Unit with project number 2015.08.23.967, and the supplies and inventories for project 2015.08.23.968 were used.)

\section{References}

[1]Back SA, Rivkees SA. Emerging concepts in periventricular white matter injury. Semin Perinatol. 2004; 28(6):405-14.

[2]Dammann O, Leviton A. Maternal intrauterine infection, cytokines, and brain damage in the preterm newborn. Pediatr Res. 1997; 42(1):1-8.

[3]Yoon BH, Jun JK, Romero R, Park KH, Gomez R, Choi JH et al. Amniotic fluid inflammatory cytokines (interleukin-6, interleukin-1beta, and tumor necrosis 
factor-alpha), neonatal brain white matter lesions, and cerebral palsy. Am J Obstet Gynecol. 1997; 177(1):19-26.

[4]Lee Y, Jung JC, Jang S, Kim J, Ali Z, Khan IA et al. Anti-Inflammatory and Neuroprotective Effects of Constituents Isolated from Rhodiola rosea. Evid Based Complement Alternat Med. 2013;2013:514049.

[5]Zaghloul N, Ahmed M. Pathophysiology of periventricular leukomalacia: What we learned from animal models. Neural Regen Res. 2017; 12(11):1795-96.

[6]Chen CH, Chan HC, Chu YT, Ho HY, Chen PY, Lee TH et al. Antioxidant activity of some plant extracts towards xanthine oxidase, lipoxygenase and tyrosinase. Molecules. 2009; 14(8):2947-58.

[7] Yu S, Liu M, Gu X, Ding F. Neuroprotective effects of salidroside in the PC12 cell model exposed to hypoglycemia and serum limitation. Cell Mol Neurobiol. 2008; 28(8):1067-78.

[8]Chen X, Liu J, Gu X, Ding F. Salidroside attenuates glutamate-induced apoptotic cell death in primary cultured hippocampal neurons of rats. Brain Res. 2008; 1238:189198.

[9]Zhao HB, Ma H, Ha XQ, Zheng P, Li XY, Zhang $M$ et al. Salidroside induces rat mesenchymal stem cells to differentiate into dopaminergic neurons. Cell Biol Int. 2014 Apr;38(4):462-71.

[10]Tuzun F, Gencpinar P, Ozbal S, Dilek M, Ergur BU, Duman N et al. Neuroprotective effect of neotrofin in a neonatal rat model of periventricular leukomalacia. Neurosci Lett. 2012;520(1):6-10.

[11]Guan S, Xiong Y, Song B, Song Y, Wang $\mathrm{D}$, Chu $\mathrm{X}$ et al. Protective effects of salidroside from Rhodiola rosea on LPSinduced acute lung injury in mice.
Immunopharmacol Immunotoxicol. 2012; 34(4):667-72.

[12] Cai Z, Pan ZL, Pang Y, Evans OB, Rhodes PG. Cytokine induction in fetal rat brains and brain injury in neonatal rats after maternal lipopolysaccharide administration. Pediatr Res. 2000; 47(1):6472.

[13] Kumral A, Baskin H, Yesilirmak DC, Ergur BU, Aykan S, Genc S et al. Erythropoietin attenuates lipopolysaccharide-induced white matter injury in the neonatal rat brain. Neonatology. 2007; 92(4):269-78.

[14]Bell MJ, Hallenbeck JM. Effects of intrauterine inflammation on developing rat brain. J Neurosci Res. 2002; 70(4):570-79. 